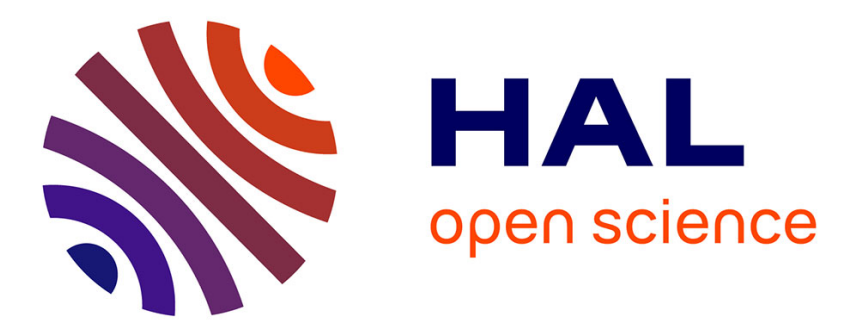

\title{
Secondary resistance to sorafenib in two patients with acute myeloid leukemia (AML) harboring FLT3-ITD mutations
}

Sebastian Scholl, Baerbel Spies-Weisshart, Anne Klink, Lars-Olof Muegge, Hans-Joerg Fricke, Andreas Hochhaus

\section{To cite this version:}

Sebastian Scholl, Baerbel Spies-Weisshart, Anne Klink, Lars-Olof Muegge, Hans-Joerg Fricke, et al.. Secondary resistance to sorafenib in two patients with acute myeloid leukemia (AML) harboring FLT3-ITD mutations. Annals of Hematology, 2010, 90 (4), pp.473-475. 10.1007/s00277-010-1027-9 . hal-00583550

\section{HAL Id: hal-00583550 https://hal.science/hal-00583550}

Submitted on 6 Apr 2011

HAL is a multi-disciplinary open access archive for the deposit and dissemination of scientific research documents, whether they are published or not. The documents may come from teaching and research institutions in France or abroad, or from public or private research centers.
L'archive ouverte pluridisciplinaire HAL, est destinée au dépôt et à la diffusion de documents scientifiques de niveau recherche, publiés ou non, émanant des établissements d'enseignement et de recherche français ou étrangers, des laboratoires publics ou privés. 


\section{Editorial Manager(tm) for Annals of Hematology}

Manuscript Draft

Manuscript Number: AOHE-D-10-00121R1

Title: Secondary resistance to sorafenib in two patients with acute myeloid leukemia (AML) harbouring FLT3-ITD mutations

Article Type: Letter to the Editor

Keywords: AML, sorafenib, FLT3-ITD, resistance

Corresponding Author: Dr. Sebastian Scholl, PD Dr. med.

Corresponding Author's Institution: Universitätsklinikum Jena

First Author: Sebastian Scholl, PD Dr. med.

Order of Authors: Sebastian Scholl, PD Dr. med.; Bärbel Spies-Weißhart, PhD; Anne Klink; Lars-Olof Mügge; Hans-Joerg Fricke; Andreas Hochhaus

Abstract: Dear Editor, we report on two patients with acute myeloid leukemia (AML) initially presenting in 2007. Both patients were tested positive for a FLT3-ITD mutation located in the juxtamembrane domain and were therefore treated with the FLT3 tyrosine kinase inhibitor sorafenib (400 mg/ day) after progress of AML following several protocols of conventional chemotherapy. Both patients responded with a significant reduction of peripheral blast counts after 10 to 17 days leading to hematological response for 12 and 14 weeks, respectively. At the time of progression, molecular analysis investigating mutations of both tyrosine kinase domains by sequencing of each individual FLT3-ITD cDNA did not demonstrate any additional mutations. We therefore suggest that the secondary resistance to sorafenib is mediated by other mechanisms than the acquisition of secondary mutations of FLT3 in these patients.

Response to Reviewers: Dear Professor Ganser,

thank you and the reviewer(s) very much for reviewing our Letter to the Editor entitled „Secondary resistance to sorafenib in two patients with acute myeloid leukemia (AML) harbouring FLT3-ITD mutations".

We carefully read the reviewer's recommendations and would like to explain our changes point by point.

1.) In patient 1 , there was a new translocation $t(9 ; 18)$ detectable at early relapse as reported on page 3. After this, there were no further cytogenetic aberrations of AML blasts in patient 1 until progression following sorafenib treatment. In contrast, in patient 2 who initially presented with a partial monosomy 7 we could only detect leukemic blasts without chromosomal aberrations prior to start of sorafenib. These data are now introduced in the paper.

2.) In the meanwhile, we also cloned and sequenced the genomic region containing the internal tandem duplication. The sequencing data confirm that both patients carried a FLT3-ITD located in the JM domain and not in the TKD1 region. 
3.) Unfortunately, we did not measure plasma concentrations of sorafenib or even intracellular reduction of STAT5 phosphorylation in the blasts of these patients.

4.) The FLT3mut/ wildtype ratio was determined in patient 1 (ratio 0,729 ) as well as in patient 2 at diagnosis (ratio 0,694). Indeed, in patient 1 there was a secondary loss of the FLT3 wildtype allele during AML progression. These aspects are now introduced in the manuscript.

5.) Indeed, the benefit of figure $2 \mathrm{C}$ is limited. We agree with the reviewer and introduced a schematic presentation of the receptor while the primer sequences are now indicated in the text.

6.) The term „relapse" was replaced by the term „progression".

7.) We changed the spelling between British and American English.

We would like to submit our revised version and would be happy if our manuscript could be considered for publication in Annals of Hematology.

Yours sincerely,

Sebastian Scholl on behalf of the authors 
*Conflict of interest
Click here to downloa

Click here to download Conflict of interest: Conflictofinterest_030310.doc 


\title{
Secondary resistance to sorafenib in two patients with acute myeloid
} leukemia (AML) harbouring FLT3-ITD mutations

\author{
Sebastian Scholl", Baerbel Spies-Weisshart, Anne Klink, Lars-Olof Muegge, Hans-Joerg \\ Fricke, Andreas Hochhaus \\ Department of Hematology and Oncology, Universitätsklinikum Jena, Germany
}

\author{
Address correspondence to: \\ PD Dr. Sebastian Scholl \\ Department of Hematology/ Oncology, Universitätsklinikum Jena \\ Erlanger Allee 101 \\ 07740 Jena \\ Germany \\ Phone: $\pm 49-3641-9324573$ \\ Fax: $\quad$ +49-3641-9324202 \\ sebastian.scholl@med.uni-jena.de
}

Running Title: Resistance to s orafenib in Flt3-ITD positive AML

Key Words: AML, sorafenib, FLT3-ITD, resistance

Word Count: 1279 


\section{Dear Editor,}

we report on two patients with acute myeloid leukaemia (AML) initially presenting in 2007. Both patients were tested positive for the FLT3-ITD mutation and were therefore treated with the FLT3 tyrosine kinase inhibitor sorafenib (400 mg/ day) after progress of AML following several protocols of conventional chemotherapy. Both patients responded with a significant reduction of peripheral blast counts after 10 to 17 days leading to hematological response for 12 and 14 weeks, respectively. At the time of relapse, molecular analysis investigating mutations of both tyrosine kinase domains by sequencing of each individual FLT3-ITD cDNA did not demonstrate any additional mutations. We therefore suggest that the secondary resistance to sorafenib is mediated by other mechanisms than the acquisition of secondary mutations of FLT3 in these patients.

FLT3-ITD mutations represent the second most frequent molecular aberration in AML leading to a constitutive activity of the class III receptor tyrosine kinase FLT3. FLT3-ITD mutations can be found in $25-30 \%$ of all AML patients and are associated with a reduced disease free survival and overall survival [1-3].

The biaryl urea sorafenib (BAY 43-9006, Bayer Schering, Leverkusen, Germany) that has been approved for the treatment of several solid tumors, e.g. renal and hepatocellular carcinoma, is able to significantly inhibit FLT3 including activating FLT3 mutations like FLT3-ITD at a nanomolar concentration [4, 5]. Furthermore, in vitro analysis of several FLT3 inhibitors revealed a different spectrum of additional mutations that may confer resistance to these inhibitors [6]. Recently, several clinical studies investigating the feasibility and efficacy of sorafenib in a small cohort of patients with relapsed FLT3-ITD positive AML have been reported including a case report demonstrating even a molecular complete remission following sorafenib treatment in a patient with AML who relapsed after allogeneic stem cell transplantation [5, 7-9]. So far, there is no report on analyses of FLT3-ITD kinase domain sequences in patients with progress of AML after treatment with sorafenib.

Patient \#1 was a 59-year-old man with AML M2 according to the French American British (FAB) classification presenting with a hyperleukocytosis at primary diagnosis. Cytogenetic and molecular genetic analyses revealed a normal karyotype and the presence of a FLT3-ITD as well as a NPM1 mutation. The patient received an induction chemotherapy consisting of idarubicine and intermediate-dosed cytarabine resulting in a complete hematologic remission of AML. Consolidation chemotherapy consisted of three courses with alternating anthracyclines (idarubicine versus mitoxantrone) combined with intermediate-dosed 
cytarabine due to a lack of availability of a sibling or unrelated donor at this time point. Only three month after consolidation therapy has been completed, disease reoccured not only in the bone marrow but also as meningeosis leukemica. Furthermore, karyotype analysis demonstrated a translocation $\mathrm{t}(9 ; 18)$ at early relapse. This patient was subsequently treated with high-dosed cytarabine and cyclophosphamide and received an additional intrathecal therapy. After the first course of this salvage protocol a partial remission could be documented while there was a second progress of AML shortly after hematological recovery following the second course of high-dosed cytarabine and cyclophosphamide. In the absence of an appropriate donor, the patient subsequently received several lines of conventional cytoreductive chemotherapies without any evidence of disease control. In this situation treatment with sorafenib (200 mg b.i.d.) was started. The patient obtained a hematological response (HR) demonstrated by a delayed clearance of peripheral blasts while there was no objective remission in the bone marrow. In detail, WBC declined from $38 / \mathrm{nl}$ prior to the commencement of sorafenib to $2.9 / \mathrm{nl}$ at day 17 and $4.2 / \mathrm{nl}$ at day 26. Contemporaneously, the percentage of peripheral blasts dropped-down from $66 \%$ to $14 \%$ at day 17 and $5 \%$ at day 26, respectively (Figure 1). Debulking of leukemia was associated with persistent anemia and thrombocytopenia. After 88 days of sorafenib treatment, a further progress of AML was observed demonstrated by rapidly increasing WBC counts including the reappearance of a high percentage of peripheral blasts. Later on, the patient died of a cerebral hemorrhage.

Patient \#2 was a 69-year-old male patient AML (FAB M2) diagnosed in 2007. At diagnosis, AML presented with $24 \%$ bone marrow blasts and signs of dyplasia. These morphological aspects and the result of cytogenetic analysis (del7q) prompted the assumption of an antecedent myelodysplastic syndrome. The patient underwent an induction chemotherapy consisting of mitoxantrone and intermediate-dosed cytarabine resulting in a complete remission. After two courses of consolidation therapy (mitoxantrone and intermediate-dosed cytarabine) persistent complete hematological remission was demonstrated. Due to the unfavourable karyotype, preparation for an allogeneic stem cell transplantation including a reduced intensity conditioning was initiated. However, the patient unfortunately relapsed within two months after completion of the consolidation therapy. While there was no severe co-morbidity in this patient, conventional chemotherapy was continued using an age-adapted regimen containing cytarabine and cyclophosphamide but bone marrow blasts persisted. Thus, we did not pursuit a curative concept any longer and palliative cytoreductive chemotherapy was started. After failure of three consecutive cytoreductive regimens treatment with sorafenib (200 mg b.i.d.) was commenced. This patient obtained HR as shown by a 
continuously decreasing number of peripheral blasts while there was no objective remission in $1.1 / \mathrm{nl}$ at day 16 while a significant reduction of the proportion of peripheral blasts was observed (from $67 \%$ to $5 \%$ ). Importantly, this patient had a persistent transfusion dependent anemia and thrombocytopenia. Treatment with sorafenib resulted in a stable HR for 101 days. At this time, WBC as well as peripheral blasts increased reflecting a secondary resistance to sorafenib treatment. Consecutive therapy with low dose cytarabine only led to a transient disease control. The patient died of a sepsis due to pneumonia and severe infectious colitis.

In both patients, a FLT3-ITD mutation was detected at diagnosis on the level of genomic DNA. We therefore confirmed the stability of these molecular aberrations before the treatment with sorafenib was started. Furthermore, FLT3-ITD expression was detected on cDNA level as well in both patients. At the time of progression during sorafenib therapy, two cDNA fragments were cloned containing the entire sequences of the tyrosine kinase domain (TKD) 1 and 2 of FLT3 in order to elucidate TKD mutations that might confer resistance to the tyrosine kinase inhibitor (Figure 2C). Since sequence analyses of several clones were not able to demonstrate any additional mutation within one of the FLT3-TKD's when both patients became refractory to sorafenib, an additional methodological approach was performed. In detail, direct sequencing of cDNA fragments with primers spanning either TKD1 or 2 did also not reveal further mutations of FLT3-ITD in both patients.

The course of these two patients treated with sorafenib shows not only the response to a monotherapy with this tyrosine kinase inhibitor after failure of different regimens with conventional chemotherapy. We can in particular demonstrate that sequence analysis after cloning of each individual FLT3-ITD allele did not reveal additional mutations within the tyrosine kinase domains. In contrast to in vitro studies with inhibitors of FLT3-ITD, we can show that apart from the acquisition of TKD mutations additional mechanisms may be responsible for the secondary resistance to sorafenib in AML patients.

Such target-independent mechanisms of resistance to tyrosine kinase inhibitors have already been demonstrated in the treatment of chronic myelogenous leukemia with imatinib. These mechanisms that are not mediated by mutations of the target gene and include clonal cytogenetic aberrations, activation of growth factor receptors, and drug influx and efflux pumps [10-13]. To our knowledge, there are no data about such mechanisms in AML patiens who were treated with a FLT3 inhibitor. Sequence analysis as demonstrated in our patients let 
us hypothesise that secondary resistance to kinase inhibitors like sorafenib might also be mediated by such target-independent mechanisms in FLT3-ITD positive AML. 


\section{References}

1. Yokota S, Kiyoi H, Nakao M, Minami S, Kuriyama K, Takeshita A, et al. (1997) Internal tandem duplication of the FLT3 gene is preferentially seen in acute myeloid leukemia and myelodysplastic syndrome among various hematological malignancies: a study on a large series of patients and cell lines. Leukemia 11:1605-1609

2. Kottaridis PD, Gale RE, Frew ME, Harrison G, Wheatley K, Groves MJ, et al. (2001) The presence of a FLT3 internal tandem duplication in patients with acute myeloid leukemia (AML) adds important prognostic information to cytogenetic risk group and response to the first cycle of chemotherapy: analysis of 854 patients from the United Kingdom Medical Research Council AML 10 and 12 trials. Blood 98:1752-1759

3. Thiede C, Steudel C, Mohr B, Schaich M, Schaekel U, Platzbecker U et al. (2002) Analysis of Flt3-activating mutations in 979 patients with acute myelogenous leukemia: association with FAB subtypes and identification of subgroups with poor prognosis. Blood 99:4326-4335.

4. Llovet JM, Ricci S, Mazzaferro V, Hilgard P, Gane E, Blanc JF, et al. (2008) Sorafenib in advanced hepatocellular carcinoma. N Engl J Med 359:378-390

5. Zhang W, Konopleva M, Shi YX, Shih YX, McQueen T, Harris D, et al. (2008) Mutant FLT3: a direct target of sorafenib in acute myelogenous leukemia. J Natl Cancer Inst 100:184-198

6. von Bubnoff N, Engh RA, Aberg E, Saenger J, Peschel C, Duyster J (2009) FMS-like tyrosine kinase 3-internal tandem duplication tyrosine kinase inhibitors display a nonoverlapping profile of resistance mutations in vitro. Cancer Res 69:3032-3041

7. Stephan Metzelder, Ying Wang, Ellen Wollmer, Michael Wanzel, Sabine Teichler, Anuhar Chaturvedi (2009) Compassionate use of sorafenib in FLT3-ITD-positive acute myeloid leukemia: sustained regression before and after allogeneic stem cell transplantation. Blood 113:6567-6571

8. Safaian NN, Czibere A, Bruns I, Fenk R, Reinecke P, Dienst A, et al. (2009) Sorafenib (Nexavar[registered]) induces molecular remission and regression of extramedullary disease in a patient with FLT3-ITD acute myeloid leukemia. Leuk Res 33:348-350

9. Mori S, Cortes J, Kantarjian H, Zhang W, Andreef M, Ravandi F (2008) Potential role of sorafenib in the treatment of acute myeloid leukemia. Leuk Lymphoma 49:2246-2255

10. Dulucq S, Bouchet S, Turcq B, Lippert E, Etienne G, Reiffers J, et al. (2008) Multidrug resistance gene (MDR1) polymorphisms are associated with major molecular responses to standard-dose imatinib in chronic myeloid leukemia. Blood 112:2024-2027 
11. White DL, Saunders VA, Dang P, Engler J, Zennettino AC, Cambareri AC, et al. (2006) OCT-1-mediated influx is a key determinant of the intracellular uptake of imatinib but not nilotinib (AMN107): reduced OCT-1 activity is the cause of low in vitro sensitivity to imatinib. Blood 108:697-704

12. Wang L, Giannoudis A, Lane S, Williamson P, Pirmohamed M, Clark RE (2008) Expression of the uptake drug transporter hOCT1 is an important clinical determinant of the response to imatinib in chronic myeloid leukemia. Clin Pharmacol Ther 83:258-264

13. La Rosée P, Hochhaus A (2010) Molecular pathogenesis of tyrosine kinase resistance in chronic myeloid leukemia. Curr Opin Hematol 17:91-96

\section{Figure legends}

\section{Figure 1}

Time course of peripheral blood counts during sorafenib treatment in patient 1 and 2. WBC (/nl), leukemic blasts (\%), platelets (/nl), hemoglobin (mmol/l)

\section{Figure 2}

\section{Detection of FLT3-ITD and sequence analysis in AML patients refractory to sorafenib.}

FLT3-ITD could be detected by PCR on the level of genomic DNA (gDNA) as well as using cDNA (A and B, respectively) in leukaemic blasts at relapse prior to start of sorafenib treatment in both patients. M, 1kb marker, “-“, negative control (THP-1 cells), “+”, positive control (MV4-11 cells), \#1, patient 1, \#2, patient 2. C, sequence analysis to detect additional mutations were performed using cDNA isolated from AML blasts at the time of secondary resistance. Two primer pairs spanning both tyrosine kinase domains (TKD) of FLT3 are marked in bold or underlined (TKD1 and 2, respectively). 
Figure 1
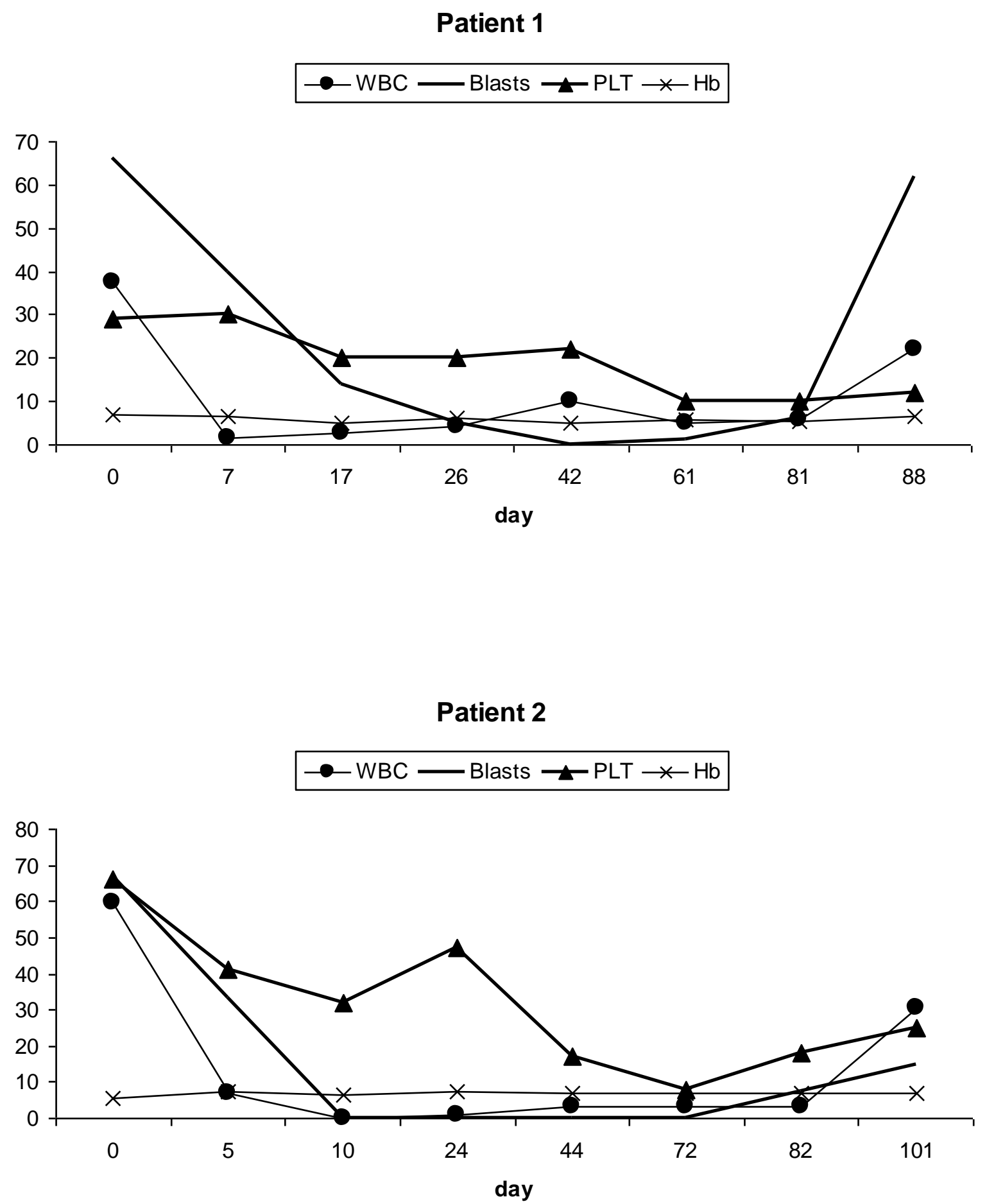


\section{Figure 2}

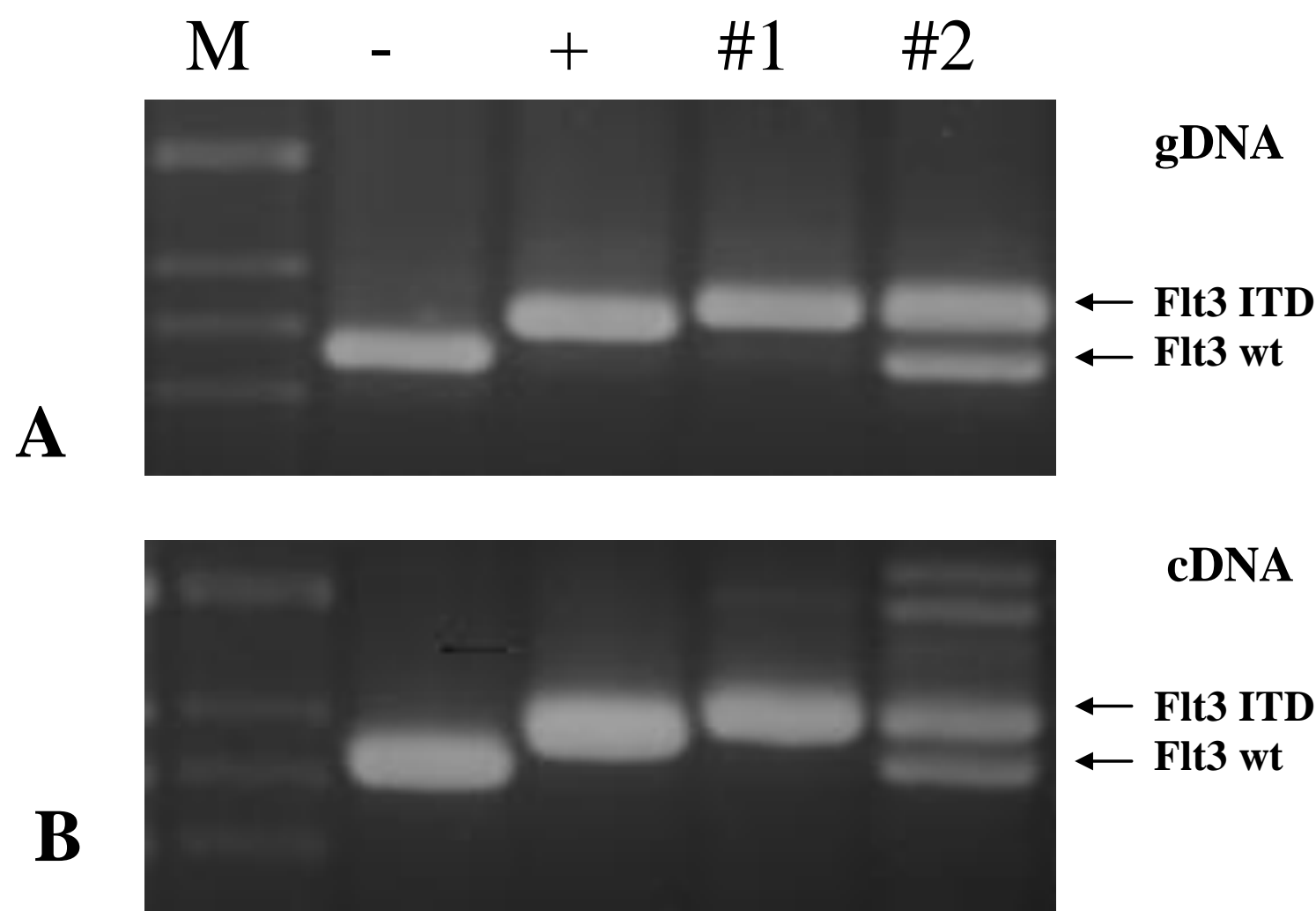

1801 aagccagcta cagatggtac aggtgaccgg ctcctcagat aatgagtact tctacgttga 1861 tttcagagaa tatgaatatg atctcaaatg ggagtttcca agagaaaat tagagtttgg 1921 gaaggtacta ggatcaggtg cttttggaaa agtgatgaac gcaacagctt atggaattag 1981 caaaacagga gtctcaatcc aggttgccgt caaaatgctg aaagaaaaag cagacagctc 2041 tgaaagagag gcactcatgt cagaactcaa gatgatgacc cagctgggaa gccacgagaa 2101 tattgtgaac ctgctggggg cgtgcacact gtcaggacca atttacttga tttttgaata 2161 ctgttgctat ggtgatcttc tcaactatct aagaagtaaa agagaaaaat ttcacaggac 2221 ttggacagag attttcaagg aacacaattt cagtttttac cccactttcc aatcacatcc 2281 aattccagc atgcctggtt caagagaagt tcagatacac ccggactcgg atcaatctc 2341 agggcttcat gggaattcat ttcactctga agatgaaatt gaatatgaaa accaaaaag 2401 gctggaagaa gaggaggact tgaatgtgct tacatttgaa gatcttcttt gctttgcata 2461 tcaagttgcc aaaggaatgg aatttctgga atttaagtcg tgtgttcaca gagacctggc 2521 cgccaggaac gtgcttgtca cccacgggaa agtggtgaag atatgtgact ttggattggc 2581 tcgagatatc atgagtgatt ccaactatgt tgtcaggggc aatgcccgtc tgcctgtaaa 2641 atggatggcc cccgaaagcc tgtttgaagg catctacacc attaagagtg atgtctggtc 2701 atatggaata ttactgtggg aatcttctc acttggtgtg aatccttacc ctggcattcc 2761 ggttgatgct aacttctaca aactgattca aaatggattt aaaatggatc agccatttta 2821 tgctacagaa gaaatataca ttataatgca atcctgctgg gcttttgact caaggaaacg 2881 gccatccttc cctaatttga cttcgtttt aggatgtcag ctggcagatg cagaagaagc 2941 gatgtatcag aatgtggatg gccgtgtttc ggaatgtcct cacacctacc aaacaggcg 


\title{
Secondary resistance to sorafenib in two patients with acute myeloid
} leukemia (AML) harbouring FLT3-ITD mutations

\author{
Sebastian Scholl ${ }^{\#}$, Baerbel Spies-Weisshart, Anne Klink, Lars-Olof Muegge, Hans-Joerg \\ Fricke, Andreas Hochhaus \\ Department of Hematology and Oncology, Universitätsklinikum Jena, Germany
}

\author{
Address correspondence to: \\ PD Dr. Sebastian Scholl \\ Department of Hematology/ Oncology, Universitätsklinikum Jena \\ Erlanger Allee 101 \\ 07740 Jena \\ Germany \\ Phone: $\pm 49-3641-9324573$ \\ Fax: $\quad$ +49-3641-9324202 \\ sebastian.scholl@med.uni-jena.de
}

Running Title: Resistance to s orafenib in Flt3-ITD positive AML

Key Words: AML, sorafenib, FLT3-ITD, resistance

Word Count: 1335 


\section{Dear Editor,}

we report on two patients with acute myeloid leukemia (AML) initially presenting in 2007. Both patients were tested positive for a FLT3-ITD mutation located in the juxtamembrane domain and were therefore treated with the FLT3 tyrosine kinase inhibitor sorafenib (400 mg/ day) after progress of AML following several protocols of conventional chemotherapy. Both patients responded with a significant reduction of peripheral blast counts after 10 to 17 days leading to hematological response for 12 and 14 weeks, respectively. At the time of progression, molecular analysis investigating mutations of both tyrosine kinase domains by sequencing of each individual FLT3-ITD cDNA did not demonstrate any additional mutations. We therefore suggest that the secondary resistance to sorafenib is mediated by other mechanisms than the acquisition of secondary mutations of FLT3 in these patients. FLT3-ITD mutations represent the second most frequent molecular aberration in AML leading to a constitutive activity of the class III receptor tyrosine kinase FLT3. FLT3-ITD mutations can be found in $25-30 \%$ of all AML patients and are associated with a reduced disease free survival and overall survival [1-3].

The biaryl urea sorafenib (BAY 43-9006, Bayer Schering, Leverkusen, Germany) that has been approved for the treatment of several solid tumors, e.g. renal and hepatocellular carcinoma, is able to significantly inhibit FLT3 including activating FLT3 mutations like FLT3-ITD at a nanomolar concentration [4, 5]. Furthermore, in vitro analysis of several FLT3 inhibitors revealed a different spectrum of additional mutations that may confer resistance to these inhibitors [6]. Recently, several clinical studies investigating the feasibility and efficacy of sorafenib in a small cohort of patients with relapsed FLT3-ITD positive AML have been reported including a case report demonstrating even a molecular complete remission following sorafenib treatment in a patient with AML who relapsed after allogeneic stem cell transplantation [5, 7-9]. So far, there is no report on analyses of FLT3-ITD kinase domain sequences in patients with progress of AML after treatment with sorafenib.

Patient \#1 was a 59-year-old man with AML M2 according to the French American British (FAB) classification presenting with a hyperleukocytosis at primary diagnosis. Cytogenetic and molecular genetic analyses revealed a normal karyotype and the presence of a FLT3-ITD as well as a NPM1 mutation. At initial diagnosis, the allelic ratio FLT3 mutated/ wildtype was 0.729. The patient received an induction chemotherapy consisting of idarubicine and intermediate-dosed cytarabine resulting in a complete hematologic remission of AML. Consolidation chemotherapy consisted of three courses with alternating anthracyclines 
(idarubicine versus mitoxantrone) combined with intermediate-dosed cytarabine due to a lack consolidation therapy has been completed, disease reoccured not only in the bone marrow but also as meningeosis leukemica. Furthermore, karyotype analysis demonstrated a translocation $\mathrm{t}(9 ; 18)$ at early relapse. This patient was subsequently treated with high-dosed cytarabine and cyclophosphamide and received an additional intrathecal therapy. After the first course of this salvage protocol a partial remission could be documented while there was a second progress of AML shortly after hematological recovery following the second course of high-dosed cytarabine and cyclophosphamide. In the absence of an appropriate donor, the patient subsequently received several lines of conventional cytoreductive chemotherapies without any evidence of disease control. In this situation, molecular analysis could still demonstrate the FLT3-ITD mutation while the wildtype allele of FLT3 was lost. Thus, treatment with sorafenib (200 mg b.i.d.) was started. The patient obtained a hematological response (HR) demonstrated by a delayed clearance of peripheral blasts while there was no objective remission in the bone marrow. In detail, WBC declined from 38/ $\mathrm{nl}$ prior to the commencement of sorafenib to $2.9 / \mathrm{nl}$ at day 17 and $4.2 / \mathrm{nl}$ at day 26. Contemporaneously, the percentage of peripheral blasts dropped-down from $66 \%$ to $14 \%$ at day 17 and $5 \%$ at day 26, respectively (Figure 1). Debulking of leukemia was associated with persistent anemia and thrombocytopenia. After 88 days of sorafenib treatment, a further progress of AML was observed demonstrated by rapidly increasing WBC counts including the reappearance of a high percentage of peripheral blasts. Later on, the patient died of a cerebral hemorrhage.

Patient \#2 was a 69-year-old male patient with FLT3-ITD-positive (allelic ratio FLT3 mutated/ wildtype 0.694) AML (FAB M2) diagnosed in 2007. At diagnosis, AML presented with $24 \%$ bone marrow blasts and signs of dyplasia. These morphological aspects and the result of cytogenetic analysis (del7q) prompted the assumption of an antecedent myelodysplastic syndrome. The patient underwent an induction chemotherapy consisting of mitoxantrone and intermediate-dosed cytarabine resulting in a complete remission. After two courses of consolidation therapy (mitoxantrone and intermediate-dosed cytarabine) persistent complete hematological remission was demonstrated. Due to the unfavourable karyotype, preparation for an allogeneic stem cell transplantation including a reduced intensity conditioning was initiated. However, the patient unfortunately relapsed within two months after completion of the consolidation therapy. While there was no severe co-morbidity in this patient, conventional chemotherapy was continued using an age-adapted regimen containing cytarabine and cyclophosphamide but bone marrow blasts persisted. Thus, we did not pursuit 
a curative concept any longer and palliative cytoreductive chemotherapy was started. cytogenetic analyses. After failure of three consecutive cytoreductive regimens treatment with sorafenib (200 mg b.i.d.) was commenced. This patient obtained HR as shown by a continuously decreasing number of peripheral blasts while there was no objective remission in the bone marrow. In detail, WBC declined from 59/nl prior to the start of sorafenib therapy to $1.1 / \mathrm{nl}$ at day 16 while a significant reduction of the proportion of peripheral blasts was observed (from 67\% to 5\%). Importantly, this patient had a persistent transfusion dependent anemia and thrombocytopenia. Treatment with sorafenib resulted in a stable HR for 101 days. At this time, WBC as well as peripheral blasts increased reflecting a secondary resistance to sorafenib treatment. Consecutive therapy with low dose cytarabine only led to a transient disease control. The patient died of a sepsis due to pneumonia and severe infectious colitis.

In both patients, a FLT3-ITD mutation was detected at diagnosis on the level of genomic DNA. We therefore confirmed the stability of these molecular aberrations before the treatment with sorafenib was started. Furthermore, FLT3-ITD expression was detected on cDNA level as well in both patients. At the time of progression during sorafenib therapy, two cDNA fragments were cloned containing the entire sequences of the tyrosine kinase domain (TKD) 1 and 2 of FLT3 in order to elucidate TKD mutations that might confer resistance to the tyrosine kinase inhibitor (Figure 2C). Since sequence analyses of several clones were not able to demonstrate any additional mutation within one of the FLT3-TKD's when both patients became refractory to sorafenib, an additional methodological approach was performed. In detail, direct sequencing of cDNA fragments with primers spanning either TKD1 or 2 did also not reveal further mutations of FLT3-ITD in both patients.

The course of these two patients treated with sorafenib shows not only the response to a monotherapy with this tyrosine kinase inhibitor after failure of different regimens with conventional chemotherapy. We can in particular demonstrate that sequence analysis after cloning of each individual FLT3-ITD allele did not reveal additional mutations within the tyrosine kinase domains. In contrast to in vitro studies with inhibitors of FLT3-ITD, we can show that apart from the acquisition of TKD mutations additional mechanisms may be responsible for the secondary resistance to sorafenib in AML patients.

Such target-independent mechanisms of resistance to tyrosine kinase inhibitors have already been demonstrated in the treatment of chronic myelogenous leukemia with imatinib. These mechanisms that are not mediated by mutations of the target gene and include clonal 
cytogenetic aberrations, activation of growth factor receptors, and drug influx and efflux pumps [10-13]. To our knowledge, there are no data about such mechanisms in AML patiens who were treated with a FLT3 inhibitor. Sequence analysis as demonstrated in our patients let us hypothesise that secondary resistance to kinase inhibitors like sorafenib might also be mediated by such target-independent mechanisms in FLT3-ITD positive AML. 


\section{References}

1. Yokota S, Kiyoi H, Nakao M, Minami S, Kuriyama K, Takeshita A, et al. (1997) Internal tandem duplication of the FLT3 gene is preferentially seen in acute myeloid leukemia and myelodysplastic syndrome among various hematological malignancies: a study on a large series of patients and cell lines. Leukemia 11:1605-1609

2. Kottaridis PD, Gale RE, Frew ME, Harrison G, Wheatley K, Groves MJ, et al. (2001) The presence of a FLT3 internal tandem duplication in patients with acute myeloid leukemia (AML) adds important prognostic information to cytogenetic risk group and response to the first cycle of chemotherapy: analysis of 854 patients from the United Kingdom Medical Research Council AML 10 and 12 trials. Blood 98:1752-1759

3. Thiede C, Steudel C, Mohr B, Schaich M, Schaekel U, Platzbecker U et al. (2002) Analysis of Flt3-activating mutations in 979 patients with acute myelogenous leukemia: association with FAB subtypes and identification of subgroups with poor prognosis. Blood 99:4326-4335.

4. Llovet JM, Ricci S, Mazzaferro V, Hilgard P, Gane E, Blanc JF, et al. (2008) Sorafenib in advanced hepatocellular carcinoma. N Engl J Med 359:378-390

5. Zhang W, Konopleva M, Shi YX, Shih YX, McQueen T, Harris D, et al. (2008) Mutant FLT3: a direct target of sorafenib in acute myelogenous leukemia. J Natl Cancer Inst 100:184-198

6. von Bubnoff N, Engh RA, Aberg E, Saenger J, Peschel C, Duyster J (2009) FMS-like tyrosine kinase 3-internal tandem duplication tyrosine kinase inhibitors display a nonoverlapping profile of resistance mutations in vitro. Cancer Res 69:3032-3041

7. Stephan Metzelder, Ying Wang, Ellen Wollmer, Michael Wanzel, Sabine Teichler, Anuhar Chaturvedi (2009) Compassionate use of sorafenib in FLT3-ITD-positive acute myeloid leukemia: sustained regression before and after allogeneic stem cell transplantation. Blood 113:6567-6571

8. Safaian NN, Czibere A, Bruns I, Fenk R, Reinecke P, Dienst A, et al. (2009) Sorafenib (Nexavar[registered]) induces molecular remission and regression of extramedullary disease in a patient with FLT3-ITD acute myeloid leukemia. Leuk Res 33:348-350

9. Mori S, Cortes J, Kantarjian H, Zhang W, Andreef M, Ravandi F (2008) Potential role of sorafenib in the treatment of acute myeloid leukemia. Leuk Lymphoma 49:2246-2255

10. Dulucq S, Bouchet S, Turcq B, Lippert E, Etienne G, Reiffers J, et al. (2008) Multidrug resistance gene (MDR1) polymorphisms are associated with major molecular responses to standard-dose imatinib in chronic myeloid leukemia. Blood 112:2024-2027 
11. White DL, Saunders VA, Dang P, Engler J, Zennettino AC, Cambareri AC, et al. (2006) OCT-1-mediated influx is a key determinant of the intracellular uptake of imatinib but not nilotinib (AMN107): reduced OCT-1 activity is the cause of low in vitro sensitivity to imatinib. Blood 108:697-704

12. Wang L, Giannoudis A, Lane S, Williamson P, Pirmohamed M, Clark RE (2008) Expression of the uptake drug transporter hOCT1 is an important clinical determinant of the response to imatinib in chronic myeloid leukemia. Clin Pharmacol Ther 83:258-264

13. La Rosée P, Hochhaus A (2010) Molecular pathogenesis of tyrosine kinase resistance in chronic myeloid leukemia. Curr Opin Hematol 17:91-96 


\section{Figure legends}

\section{Figure 1}

Time course of peripheral blood counts during sorafenib treatment in patient 1 and 2. WBC $(/ \mathrm{nl})$, leukemic blasts $(\%)$, platelets $(/ \mathrm{nl})$, hemoglobin $(\mathrm{mmol} / \mathrm{l})$

\section{Figure 2}

Detection of FLT3-ITD and sequence analysis in AML patients refractory to sorafenib. FLT3-ITD could be detected by PCR on the level of genomic DNA (gDNA) as well as using cDNA (A and B, respectively) in leukaemic blasts at progression prior to start of sorafenib treatment in both patients. M, 1kb marker, “-“, negative control (THP-1 cells), “+”, positive control (MV4-11 cells), \#1, patient 1, \#2, patient 2. C, Schematic illustration of FLT3 receptor tyrososine kinase with its different domains: EC, extracellular domain; TD, transmembrane domain; JM, juxtamembrane; TKD, tyrosine kinase domain; KI, kinase insert. The arrows indicate the primers used for cloning TKD1 (TKD1fw GACCGGCTCCTCAGATAATGAG, TKD1rv CAGGTCTCTGTGAACACACGAC) and TKD2 (TKD1fw TCCAGCATGCCTGGTTCAAGAG, TKD1rv GTGAGGACATTCCGAAACAC), respectively. 
Figure 1

\section{Patient 1}

$\multimap$ WBC $\longrightarrow$ Blasts $\leftarrow$ PLT $\leftarrow \mathrm{Hb}$

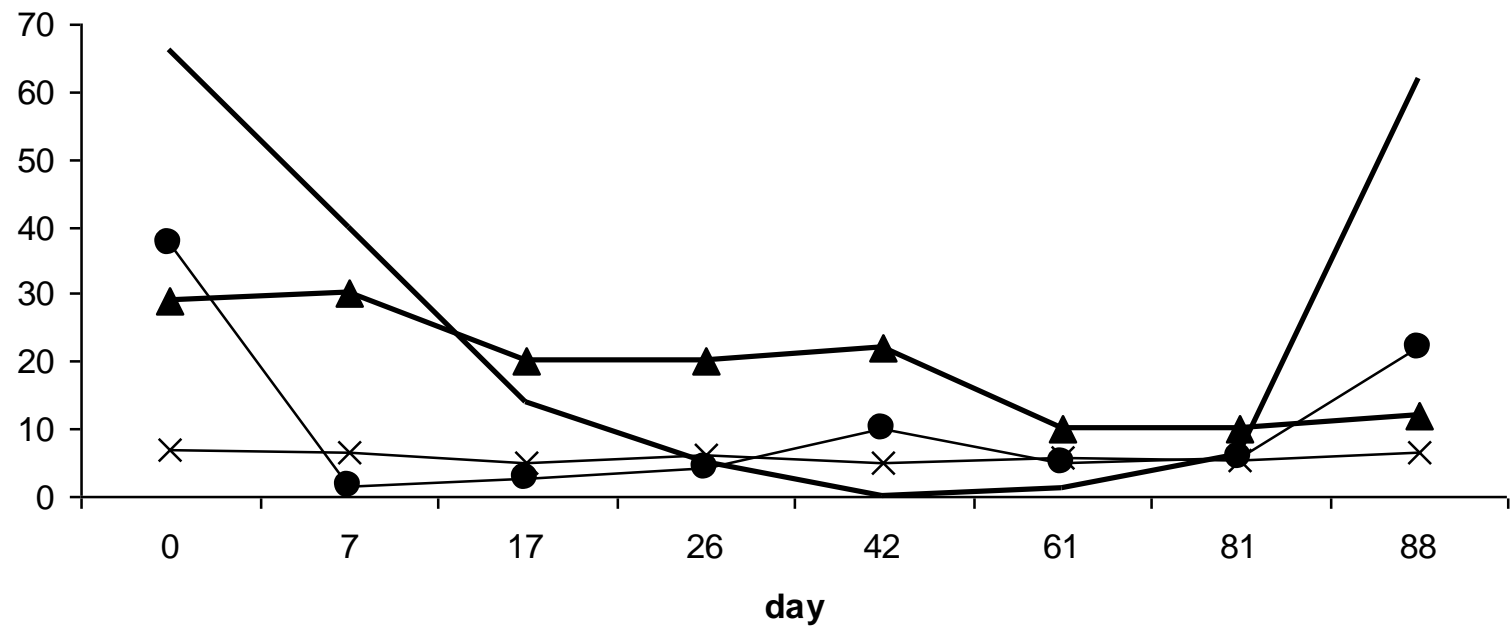

\section{Patient 2}

$\rightarrow$ WBC $\longrightarrow$ Blasts $\leftarrow$ PLT $\leftarrow \mathrm{Hb}$

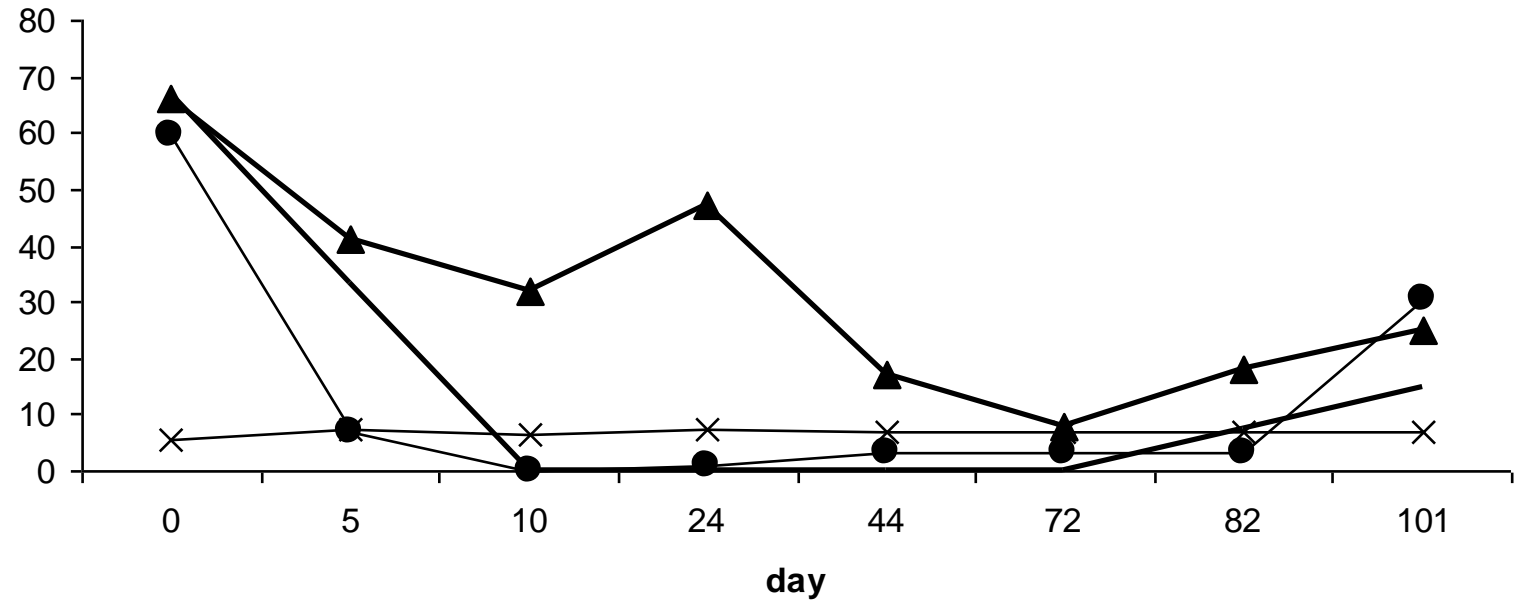


Figure 2
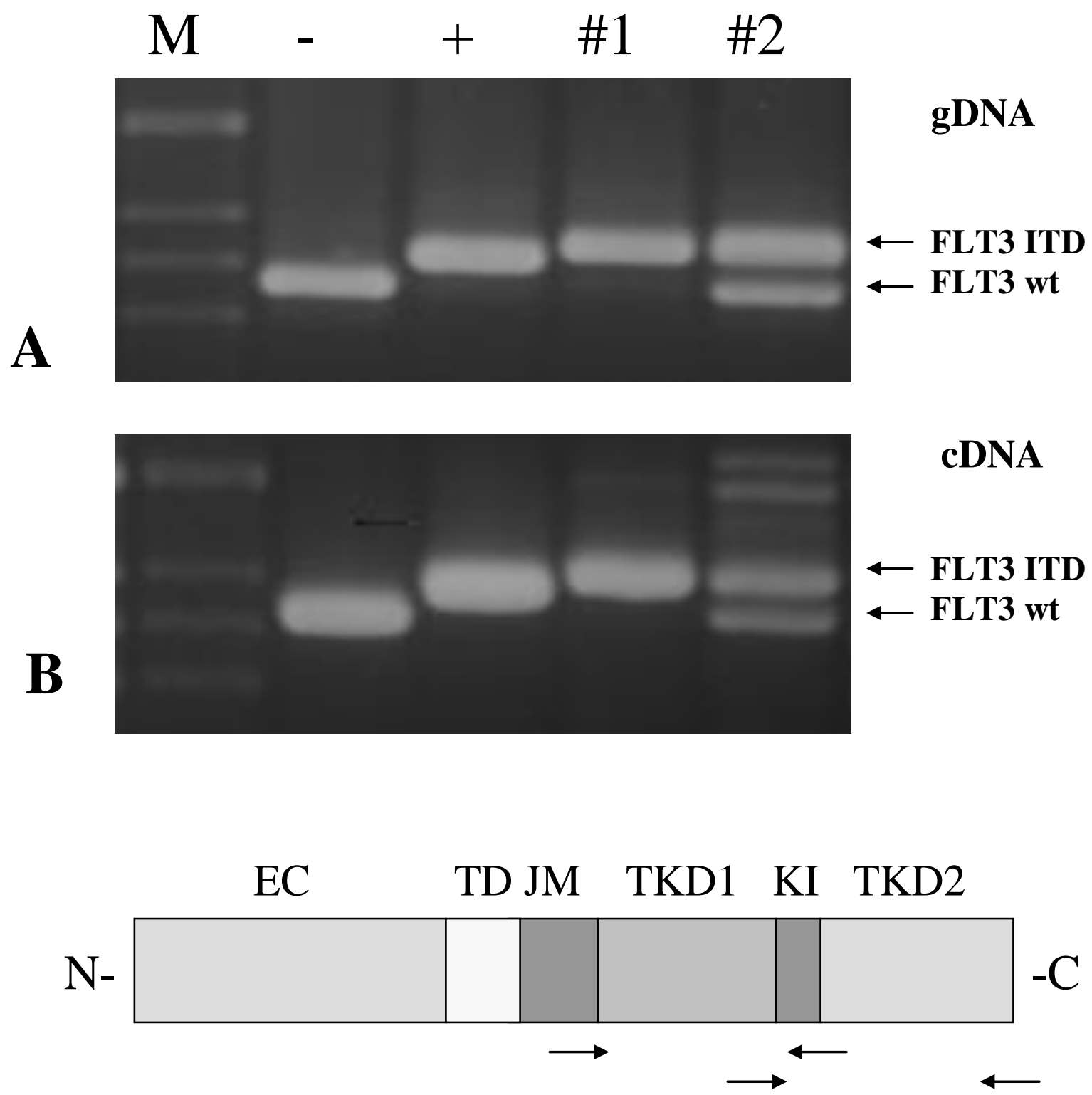

C 